\title{
Complicaciones de la linfadenectomía retroperitoneal postquimioterapia en pacientes con tumor germinal de origen testicular: Experiencia de 12 años en un centro de referencia en Colombia
}

\section{Post-chemotherapy Retroperitoneal Lymphadenectomy Complications in Patients with Testicular Germ Cell Tumors: 12-Year Experience at a Reference Center in Colombia}

\author{
Lynda Torres ${ }^{10}$ José Gustavo Ramos ${ }^{2}$ Rodolfo Varela ${ }^{2}$ Marino Cabrera ${ }^{2}$ Paola Andrea Orrego ${ }^{3}$
} Aysa Vanessa Mosquera ${ }^{3}$ Sandra Viviana Pulido Vecino 4

\footnotetext{
1 Pontificia Universidad Javeriana, Centro Policlínico Olaya, Bogotá, Colombia

2 Instituto Nacional de Cancerología, Bogotá, Colombia

3 Universidad Militar Nueva Granada, Bogotá, Colombia

${ }^{4}$ Universidad Militar Nueva Granada, Bogotá, Colombia
}

Address for correspondence Lynda Torres Castellanos, MD, PontificiaUniversidad Javeriana, Carrera 7 \# 40-62, Bogotá, 111511 , Colombia (e-mail: lynda.torresc@gmail.com).

\section{Resumen}

\section{Palabras clave}

- neoplasia residual

- tumor retroperitoneal

- tumor germinal

- resección de ganglios linfáticos

- complicaciones
Objetivo El objetivo de este estudio, fue describir las complicaciones intraoperatorias y postoperatorias, así como la necesidad de cirugías concomitantes en la linfadenectomía retroperitoneal postquimioterapia en un centro de referencia de manejo de cáncer.

Métodos Se recolectaron datos de una cohorte retrospectiva de pacientes con diagnóstico de tumor germinal de origen testicular que hubiesen recibido quimioterapia y en quienes se documentó tumor residual retroperitoneal y fueron sometidos a LRP-PC durante 12 años en un centro de referencia de manejo de cáncer. Resultados Se practicó LRP-PC a 64 pacientes. La edad promedio al momento de la cirugía fue 28,1 años (18-47 años). El tamaño promedio de la masa retroperitoneal post quimioterapia fue 6,7 (1-28 cm). La estancia hospitalaria promedio fue 7,9 días (rango 1-99 días), la tasa de cirugías adicionales fue del $20 \%$. La tasa de complicaciones mayores fue de $7,8 \%$. Tener seminoma en la histología testicular inicial se asoció con un mayor sangrado y el tamaño de la masa retroperitoneal residual se asoció con la necesidad de cirugías concomitantes.

Conclusiones La LRP-PC es una cirugía de alto nivel de complejidad que se asocia a complicaciones mayores y a la necesidad de cirugías concomitantes. Esta cohorte de pacientes muestra desenlaces similares a los descritos en la literatura, recalcando el hecho de que esta cirugía, debería ser realizada en centros de referencia de manejo del cáncer. received

May 31, 2020

accepted

September 25, 2020

published online

February 4, 2021
DOI https://doi.org/

$10.1055 / \mathrm{s}-0040-1721327$.

ISSN 0120-789X.

e ISSN 2027-0119. (c) 2021. Sociedad Colombiana de Urología. All rights reserved. This is an open access article published by Thieme under the terms of the Creative Commons Attribution-NonDerivative-NonCommercial-License, permitting copying and reproduction so long as the original work is given appropriate credit. Contents may not be used for commercial purposes, or adapted, remixed, transformed or built upon. (https://creativecommons.org/ licenses/by-nc-nd/4.0/)

Thieme Revinter Publicações Ltda., Rua do Matoso 170, Rio de Janeiro, RJ, CEP 20270-135, Brazil 
Abstract

\section{Keywords}

- residual neoplasm

- retroperitoneal cancer

- germinal

- lymph node dissection

- complications
Objective The purpose was to describe complications and concomitant surgeries in PC-RPLND in a Referal cancer center.

Methods Data were collected from a retrospective cohort of patients diagnosed with a germ cell tumor of testicular origin who had received chemotherapy and they were diagnosed with retroperitoneal residual tumor and underwent PC-RPLND in a single cancer referral center during 12 years.

Results PC-RPLND was performed in 64 patients. The mean age at moment of surgery was 28.1 years (18-47 years). The mean size of the retroperitoneal residual mass was $6.7(1-28 \mathrm{~cm})$. The average hospital stay was 7.9 days (range 1-99 day), the rate of additional surgeries was $20 \%$. The major complication rate was $7.8 \%$. Seminoma histology in testicular tumor was associated with increased bleeding; the size of the residual retroperitoneal mass was associated with the need of concomitant surgeries. Conclusion PC-RPLND is a complex surgery that is associated with major complications and the need of concomitant surgeries. This research shows similar outcomes previously described in the literature highlighting the fact that this surgery should be performed in reference cancer treatment centers.

\section{Introducción}

El tumor germinal de origen testicular representa la malignidad más común en hombres entre los 15 y 35 años de edad y la incidencia se ha duplicado en los últimos 40 años. $^{1,2}$ Hasta el $30 \%$ de los pacientes pueden tener enfermedad metastásica al momento del diagnóstico. ${ }^{1}$

A mediados de los años setenta, la linfadenectomía retroperitoneal postquimioterapia (LRP-PC) era el primer método terapéutico de los pacientes con tumor germinal testicular y metástasis en el retroperitoneo, posteriormente se presentó un cambio en la secuencia de eventos y con el régimen de medicamentos de quimioterapia basados en platinos se empezó a administrar quimioterapia y posteriormente LRP-PC. ${ }^{3,4}$ Actualmente la LRP-PC está indicada en seminoma cuando la masa residual es mayor de $3 \mathrm{~cm}$ y los marcadores tumorales son negativos, adicionalmente la tomografía por emisión de positrones debe ser positiva; en tumor germinal no seminomatoso se debe realizar LRP-PC en todas las masas residuales mayores de $1 \mathrm{~cm}$ y marcadores tumorales negativos. ${ }^{5,6}$

La LRP-PC es una cirugía compleja teniendo en cuenta la aparición de factores tumorales tales como la presencia de reacción desmoplásica, escenario en donde el tumor puede causar una distorsión de los planos de disección, asociándose al compromiso de estructuras adyacentes al tumor siendo necesarios procedimientos concomitantes y resección de otras estructuras para lograr la remoción completa de la masa residual. ${ }^{7}$ Adicionalmente por la complejidad del procedimiento quirúrgico puede estar asociado a complicaciones mayores. $^{7,8}$

El objetivo de este estudio, fue describir las complicaciones intraoperatorias y postoperatorias, así como la necesidad de cirugías concomitantes en la linfadenectomía retroperitoneal postquimioterapia en un centro de referencia de manejo de cáncer durante 12 años.

\section{Métodos}

Se recolectaron datos de una cohorte retrospectiva de una única institución centro de referencia de manejo de cáncer en Colombia previa aprobación por el comité de ética de esta institución. Se incluyeron pacientes con diagnóstico de tumor germinal de origen testicular seminomatoso $y$ no seminomatoso, que hubiesen recibido quimioterapia y en quienes se documentó masa residual y fueron sometidos a LRP-PC. La muestra de este estudio fue la totalidad de los pacientes sometidos a LRP-PC desde el año 2006 hasta 2018. Todas las cirugías se realizaron mediante abordaje abierto por urólogos oncólogos con experiencia. Se obtuvieron datos entre enero de 2006 y octubre de 2018 . Los datos fueron recolectados a partir del registro en la historia clínica, informes quirúrgicos, e informes de patología y radiología. Se definió el grupo pronóstico de los pacientes prequimioterapia de acuerdo con la International Germ Cell Cancer Collaborative Group, se clasificaron en pronóstico bueno, intermedio y pobre. ${ }^{9}$ Las severidades de las complicaciones se definieron de acuerdo con la escala de clasificación de Clavien Dindo. ${ }^{10}$ Adicionalmente, se documentó la necesidad de cirugías adicionales y la estancia hospitalaria.

El análisis estadístico se realizó utilizando Stata version 15 (StataCorp, College Station, Texas). Se utilizó el test de ANOVA para la comparación de medias entre múltiples grupos, y la comparación entre dos variables categóricas se realizó mediante el test de Chi cuadrado y adicionalmente la comparación entre grupos mediante la prueba de MannWhitney. El valor de p menor o igual de 0,05 se consideró estadísticamente significativo.

\section{Resultados}

La LRP-PC se practicó a 64 pacientes. La edad promedio de los pacientes al momento de la cirugía fue 28,1 años (18-47 
Tabla 1 Características de los pacientes

\begin{tabular}{|l|l|}
\hline Variable & $\begin{array}{l}\mathrm{n}(\%) / \text { mediana } \\
(\text { Rango })\end{array}$ \\
\hline Edad años $(\mathrm{n}=64)$ & $\begin{array}{l}28,1(18-47) \\
\mathrm{DE}=6,5\end{array}$ \\
\hline Estadio clínico & $\mathrm{n}=64 \mathrm{n}(\%)$ \\
\hline EC I con recaída & $4(6,2)$ \\
\hline EC II & $39(60,9)$ \\
\hline EC III & $21(32,8)$ \\
\hline Grupo pronóstico IGCCCG NSGCT** & $(\mathrm{n}=59) \%(92,9)$ \\
\hline Bueno & $46(77,9)$ \\
\hline Intermedio & $7(11,9)$ \\
\hline Pobre & $6(10,1)$ \\
\hline Grupo pronóstico IGCCCG SGCT** & $(\mathrm{n}=5) \%(7,8)$ \\
\hline Bueno & $5(100)$ \\
\hline Intermedio & $0(0)$ \\
\hline $\begin{array}{l}\text { Tamaño de la masa retroperitoneal } \\
\text { postquimioterapia (cm) }\end{array}$ & $6,7(1-28)$ \\
\hline
\end{tabular}

*Nonseminomatous Germ Cell Tumor.

${ }^{* *}$ Seminomatous Germ Cell Tumor.

años). La mayoría de los pacientes tenían estadio clínico II y eran de grupo pronóstico bueno. Respecto a la histología testicular el $75 \%$ de los pacientes tuvieron tumor testicular no seminomatoso, $8,3 \%$ seminoma puro, $8,3 \%$ teratoma puro y $8,3 \%$ carcinoma embrionario puro (-Tabla $\mathbf{1}$ ).

Las complicaciones intraquirúrgicas mayores y la necesidad de cirugías adicionales ocurrieron en $5(8,1 \%$ ) y en 13 (20,3\%) de los pacientes respectivamente. El sangrado promedio fue 1270 mililitros y la estancia hospitalaria promedio fue de 7,9 días (rango 1-99 días). En la - Tabla 2 se muestra en detalle el número y tipo de cirugías concomitantes. Las complicaciones mayores (clasificación Clavien Dindo 4 y 5 ) ocurrieron en cinco pacientes. La única complicación Clavien Dindo 4 correspondió a un paciente que tuvo requerimiento de terapia de reemplazo renal y estancia en unidad de cuidados intensivos. Las complicaciones Clavien Dindo 5 ocurrieron en cuatro pacientes (6,2\%), en detalle, un paciente presentó broncoaspiración durante la inducción anestésica para procedimiento de cierre de la pared abdominal, el segundo paciente presentó accidente cerebrovascular, el tercer paciente

Tabla 2 Cirugías concomitantes realizadas en la linfadenectomía retroperitoneal post-quimioterapia

\begin{tabular}{|l|l|}
\hline $\begin{array}{l}\text { Cirugías concomitantes } \\
\mathbf{n}=\mathbf{1 3}, \mathbf{n}(\%) \mathbf{2 0 , 3} \%\end{array}$ & $\mathbf{n}(\%) / m e d i a n a$ (Rango) \\
\hline Nefrectomía & $7(54,0)$ \\
\hline Vascular & $3(23,0)$ \\
\hline Intestinal & $2(15,4)$ \\
\hline Resección masa pulmonar & $1(7,6)$ \\
\hline Estancia hospitalaria (días) & $7,9(1-99) \mathrm{DE}=12,6$ \\
\hline
\end{tabular}

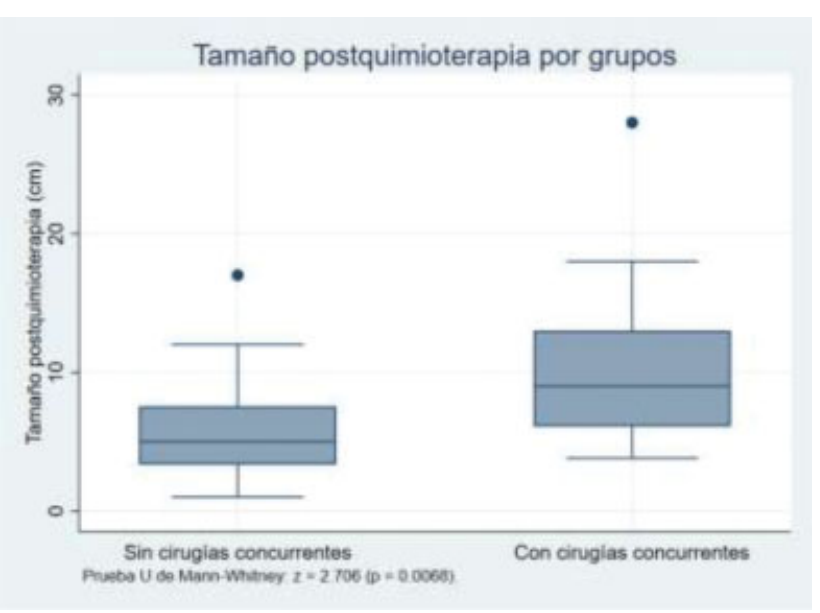

Fig. 1 Asociación del tamaño de la masa residual postquimioterapia con la necesidad de cirugías concomitantes.

presentó sepsis de origen abdominal secundaria a lesión duodenal y el cuarto paciente presentó coagulopatía en el intraoperatorio secundario a sangrado masivo.

Al documentar los posibles factores asociados a cirugías adicionales y complicaciones postquirúrgicas, se encontró que aquellos pacientes con componente de seminoma y seminoma puro en la histología testicular, presentaban mayor sangrado $(>1500 \mathrm{ml}$ ) p=0,030. Adicionalmente, el tamaño de la masa residual postquimioterapia estaba asociada con la necesidad de cirugías concomitantes, $\mathrm{p}=0,0068$. (-Figura 1 ).

\section{Discusión}

El tumor testicular corresponde al tumor maligno más común de hombres entre los 15 y los 35 años de edad ${ }^{1} \mathrm{y}$ quienes se presentan con metástasis en el retroperitoneo al momento del diagnóstico de acuerdo al valor de los marcadores tumorales, tienen la opción de recibir quimioterapia y adicionalmente si presentan masa residual postquimioterapia está la opción de manejo quirúrgico mediante linfadenectomía retroperitoneal citorreductora. ${ }^{3}$

Nuestra cohorte de pacientes confirma la complejidad del procedimiento quirúrgico en este escenario y la necesidad de intervenciones quirúrgicas adicionales relacionadas principalmente con el tamaño de la masa residual, requiriendo procedimientos como nefrectomía, cirugía vascular e intestinal, ya que en ese escenario, puede existir distorsión de los planos anatómicos, compromisos de estructuras adyacentes como la aorta, uréter y vena cava. Las cirugías concomitantes se realizaron en el 20,3\% de los pacientes y la cirugía adicional más común fue la nefrectomía. El estudio de Djaladat demostró que el 30\% de los pacientes requieren procedimientos concurrentes al momento de la linfadenectomía retroperitoneal postquimioterapia. ${ }^{7}$ Cosidine y colaboradores en el resultado de la cohorte de pacientes publicado en el 2016, demostraron que es necesario realizar cirugías adicionales en $28 \%$ de los pacientes y la más frecuente correspondió a procedimientos vasculares en el $15 \%{ }^{8}$ La incidencia de procedimientos adicionales es comparable a otros estudios, varía entre un 
$19 \%$ a un $33 \%{ }^{11,12}$ Adicionalmente, el tamaño de la masa residual postquimioterapia se asoció a la necesidad de cirugías concomitantes.

En nuestro estudio, se documentó que tener seminoma en la histología testicular se asocia a un mayor sangrado. Ese hallazgo fue documentado en el 2006 por Russell y colaboradores, quienes indicaron que a mayor porcentaje de seminoma en el testículo mayor sangrado estimado durante el procedimiento quirúrgico. ${ }^{13}$ Posiblemente siendo secundaria a la reacción desmoplásica generada después de la quimioterapia en esa histología. La estancia hospitalaria de nuestra cohorte es similar a la de otros estudios. En el estudio de Considine 2015 la estancia hospitalaria promedio fue 10,5 días, en el estudio de Heindereich 12 días y en el de Cary y col., la estancia hospitalaria documentada fue 4,4 días. La estancia hospitalaria se vio afectada al haber complicaciones asociadas al procedimiento quirúrgico. ${ }^{8,14,15}$

Esta cirugía en los últimos años en los centros de referencia ha demostrado menor incidencia de complicaciones. $^{16}$ Debido a la experiencia quirúrgica y el cuidado postoperatorio estandarizado en las instituciones de alto volumen. Ya está documentado que cuando se da el manejo en instituciones de referencia hay menor número de complicaciones respecto cuando se da el manejo en instituciones de bajo volumen de casos, lo anterior también descrito en otro tipo de neoplasias. ${ }^{17,18}$ La tasa de complicaciones mayores en nuestro estudio fue del 6,2\% explicado por la complejidad de los casos. A pesar de la naturaleza retrospectiva del estudio y del tamaño de la muestra, tiene resultados similares a los documentados en otros centros de alto volumen, adicionalmente representa la tendencia en cuanto a complicaciones y la necesidad de cirugías concomitantes y nos muestra que la LRP-PC es una de las herramientas para el manejo con intención curativa de pacientes en el escenario de masa residual postquimioterapia. Es un procedimiento de gran desafío para el urólogo y aproximadamente en el $20 \%$ de los casos se puede necesitar la participación de otras especialidades para asegurar la resección completa del tumor residual.

\section{Conclusión}

La linfadenectomía retroperitoneal post quimioterapia es parte del manejo integral en pacientes con tumor residual retroperitoneal después de quimioterapia en tumor de células germinales. Esa intervención se asocia a la necesidad de otros procedimientos quirúrgicos concomitantes y a complicaciones que pueden ser mayores. Teniendo en cuenta la complejidad de la intervención, ese procedimiento debería ser realizado en instituciones de referencia de manejo del cáncer.

\section{Aspectos Éticos}

De acuerdo a la Resolución 8430 del año 1993 (expedida por el Ministerio de Salud de Colombia), este estudio se encuentra en una categoría sin riesgo. Se tiene además en cuenta la normatividad internacional: la declaración de Helsinki y las pautas éticas para la investigación biomédica del Consejo de Organizaciones
Internacionales de las Ciencias Médicas -CIOMS- y los parámetros establecidos por la Resolución 8430 de 1993 y Resolución 2378 de 2008.

El presente estudio es retrospectivo y no se realizó ningún tipo de intervención, ni modificación intencionada de variables biológicas, fisiológicas, psicológicas o sociales de los participantes. Se realizó revisión de historias clínicas para la recolección de los datos. Se garantiza la confidencialidad de los datos recolectados y los resultados por parte de todos los investigadores. Así mismo, es un estudio sin implicaciones ambientales. Este estudio fue presentado al comité de ética del Instituto Nacional de Cancerología.

\section{Conflicto de Intereses}

Los autores declaran no tener ningún conflicto de intereses.

\section{Referencias}

1 Le Cornet C, Lortet-Tieulent J, Forman D, et al. Testicular cancer incidence to rise by $25 \%$ by 2025 in Europe? Model-based predictions in 40 countries using population-based registry data. Eur J Cancer 2014;50(04):831-839

2 Bray F, Ferlay J, Soerjomataram I, Siegel RL, Torre LA, Jemal A. Global cancer statistics 2018: GLOBOCAN estimates of incidence and mortality worldwide for 36 cancers in 185 countries. CA Cancer J Clin 2018;68(06):394-424

3 Rowland R, Donohue J. Cytoreductive Surgery in Testicular Cancer. World J Urol 1984;2:48-52

4 Donohue JP, Einhorn LH, Williams SD. Cytoreductive surgery for metastatic testis cancer: considerations of timing and extent. J Urol 1980;123(06):876-880

5 Albers P, Albrecht W, Algaba F, et al; European Association of Urology. Guidelines on Testicular Cancer: 2015 Update. Eur Urol 2015;68(06):1054-1068

6 Motzer RJ, Jonasch E, Agarwal N, et al. Testicular Cancer, Version 2.2015. J Natl Compr Canc Netw 2015;13(06):772-799

7 Djaladat H, Nichols C, Daneshmand S. Adjuvant surgery in testicular cancer patients undergoing postchemotherapy retroperitoneal lymph node dissection. Ann Surg Oncol 2012; 19(07):2388-2393

8 Considine S, Heaney R, Conroy R, Thornhill JA. Post-chemotherapy retroperitoneal lymph node dissection in the management of metastatic testis cancer: the 16-year experience in an Irish setting. Ir J Med Sci 2016;185(04):901-907

9 International Germ Cell Cancer Collaborative Group. International Germ Cell Consensus Classification: a prognostic factor-based staging system for metastatic germ cell cancers. J Clin Oncol 1997;15(02):594-603

10 Clavien PA, Barkun J, de Oliveira ML, et al. The Clavien-Dindo classification of surgical complications: five-year experience. Ann Surg 2009;250(02):187-196

11 Heidenreich A, Pfister D, Witthuhn R, Thüer D, Albers P. Postchemotherapy retroperitoneal lymph node dissection in advanced testicular cancer: radical or modified template resection. Eur Urol 2009;55(01):217-224

12 Winter C, Pfister D, Busch J, et al. Residual tumor size and IGCCCG risk classification predict additional vascular procedures in patients with germ cell tumors and residual tumor resection: a multicenter analysis of the German Testicular Cancer Study Group. Eur Urol 2012;61(02):403-409

13 Russell CM, Sharma P, Agarwal G, et al. Is percent seminoma associated with intraoperative morbidity during postchemotherapy RPLND? Can J Urol 2016;23(01):8127-8134 
116 Complicaciones de la linfadenectomía retroperitoneal postquimioterapia Torres y col.

14 Heidenreich A, Haidl F, Paffenholz P, Pape C, Neumann U, Pfister D. Surgical management of complex residual masses following systemic chemotherapy for metastatic testicular germ cell tumours. Ann Oncol 2017;28(02):362-367

15 Cary C, Masterson TA, Bihrle R, Foster RS. Contemporary trends in postchemotherapy retroperitoneal lymph node dissection: Additional procedures and perioperative complications. Urol Oncol 2015;33(09):389.e15-389.e21

16 Mosharafa AA, Foster RS, Koch MO, Bihrle R, Donohue JP. Complications of post-chemotherapy retroperitoneal lymph node dissection for testis cancer. J Urol 2004;171(05):1839-1841

17 Hillner BE, Smith TJ, Desch CE. Hospital and physician volume or specialization and outcomes in cancer treatment: importance in quality of cancer care. J Clin Oncol 2000;18(11):2327-2340

18 Correa JJ, Velásquez D, Ramiro A, et al. Testicular cancer colombian guideline. Urol Colomb. 2016;25(03):274-285 